\title{
Building high-performance integrated optical devices using subwavelength grating metamaterials -INVITED
}

\author{
Alejandro Sánchez-Postigo ${ }^{1, *}$, Pablo Ginel-Moreno ${ }^{1}$, Alejandro Ortega-Moñux ${ }^{1}, J$. Gonzalo Wangüemert-Pérez ${ }^{1}$, Robert \\ Halir ${ }^{1}$, Daniel Pereira-Martín ${ }^{1}$, Abdelfettah Hadij-ElHouati ${ }^{1}$, Jens H. Schmid ${ }^{2}$, Shurui Wang ${ }^{2}$, Martin Vachon ${ }^{2}$, Dan-Xia \\ $\mathrm{Xu}^{2}$, Winnie N. Ye ${ }^{3}$, Jordi Soler Penadés ${ }^{4,5}$, Milos Nedeljkovic ${ }^{4}$, Goran Z. Mashanovich ${ }^{4}$, Pavel Cheben ${ }^{2}$, and Ínigo \\ Molina-Fernández ${ }^{1}$ \\ ${ }^{1}$ Telecommunication Research Institute (TELMA), Universidad de Málaga, CEI Andalucía TECH, Louis Pasteur 35, 29010 Málaga, \\ Spain \\ ${ }^{2}$ National Research Council Canada, 1200 Montreal Road, Bldg. M50, Ottawa K1A 0R6, Canada \\ ${ }^{3}$ Department of Electronics, Carleton University, 1125 Colonel By Drive, Ottawa, Ontario K1S5B6, Canada \\ ${ }^{4}$ Optoelectronics Research Centre, University of Southampton, Southampton, SO17 1BJ, United Kingdom \\ ${ }^{5}$ Currently at VLC Photonics S.L., Valencia, Spain
}

\begin{abstract}
The use of subwavelength grating structures in silicon waveguides have fuelled the development of integrated optical components with superior performance. By a judicious lithographic patterning of the grating, the optical properties of the synthesized metamaterial can be accurately tailored. In this work, we review our latest advances in subwavelength-grating-engineered silicon and germanium planar devices.
\end{abstract}

\section{Introduction}

Subwavelength grating (SWG) metamaterials are becoming established as an advantageous tool to manipulate light in integrated photonic devices [1-3]. SWG nanophotonic waveguides comprise an arrangement of structures with a period $(\Lambda)$ that is smaller than half the effective wavelength in the waveguide (i.e., $\Lambda<0.5 \lambda / n_{\mathrm{B}}$, where $n_{\mathrm{B}}$ is the effective index of the fundamental BlochFloquet mode that is guided). Under this condition, the SWG structure behaves as an anisotropic homogeneous artificial material. The optical properties of the SWG waveguide, including the refractive index contrast and the wavelength dispersion, can be controlled by choosing the geometrical parameters (e.g., period and duty cycle) of the lithographically defined patterns. In this work, we leverage the refractive-index engineering possibilities of SWG metamaterials to implement high-performance waveguide-based devices for the near- and mid-infrared wavelength ranges.

\section{Broadband surface grating couplers with sub-decibel coupling efficiency}

Efficient and broadband fibre-to-chip coupling is a major challenge in silicon photonics [4]. To address this problem, surface grating couplers are often used, as they allow wafer-scale testing and provide robust misalignment tolerances. These devices are diffractive, periodic waveguide structures that satisfy the phase matching condition between a guided Bloch-Floquet mode and a free-propagating off-chip plane wave, as described by [5]

$$
n_{\mathrm{a}} \sin (\theta)=n_{\mathrm{B}}+m \frac{\lambda}{\Lambda},
$$

where $n_{\mathrm{a}}$ is the refractive index of the medium to which the leaky Bloch-Floquet mode is radiated, $\theta$ is the radiation angle, $n_{\mathrm{B}}$ is the effective index of the mode, $m$ is the radiation order, $\lambda$ is the operating wavelength, and $\Lambda$ is the grating period. To collect most radiated power, an optical fibre is conveniently positioned, with a given tilt angle, above the surface grating coupler.

The two important parameters in surface grating couplers are the coupling efficiency (CE) and the 1-dB bandwidth (BW). For a given fibre mode field diameter, there is a trade-off between these two parameters and the coupling efficiency-bandwidth product (CE-BW) is an important figure of merit. We have recently shown how to break the coupling efficiency-bandwidth product limit of surface grating couplers by using SWG metamaterials and a silicon prism to radiate in the zeroth diffraction order [6].

Our proposed zero-order grating coupler, illustrated in Fig. 1(a), consists of an SWG waveguide of pitch $\Lambda$ and duty cycle $\mathrm{DC}=a / \Lambda$. By operating in the subwavelength regime, all diffraction orders are frustrated. However, by positioning a silicon prism $\left(n_{\mathrm{a}} \sim 3.45>n_{\mathrm{B}}\right)$ on top of the subwavelength grating, radiation is enabled for $m=0$. Therefore, in a ZGC, the phase matching condition becomes

$$
n_{\mathrm{a}} \sin (\theta)=n_{\mathrm{B}},
$$

\footnotetext{
${ }^{*}$ Corresponding author: asp@ic.uma.es
} 
where the dependency between the radiation angle and the wavelength only arises from the silicon refractive index $\left[n_{\mathrm{a}}=n_{\mathrm{a}}(\lambda)\right]$ and the grating effective index $\left[n_{\mathrm{B}}=n_{\mathrm{B}}(\lambda)\right]$, thus increasing the bandwidth with respect to conventional grating couplers. The radiation strength, and hence the coupling efficiency, depends on the prism angle $\phi_{\text {prism, the tilt angle }} \phi_{\text {clad }}$, and the initial thickness $t_{0}$ of the wedge-shaped air gap between the prism and the grating.

The light injected into the grating from the input waveguide is evanescently coupled to the prism and then into an SMF-28 optical fibre via an anti-reflection coated facet. As shown in Fig. 1(b), a two-stage transition at the beginning of the grating adapts the fundamental mode of the input waveguide (width $W=500 \mathrm{~nm}$ ) to the BlochFloquet mode of the grating waveguide (width $W_{\mathrm{g}}=$ $15 \mu \mathrm{m})$. This transition is composed of a $200-\mu \mathrm{m}$-long linear taper and a 3- $\mu \mathrm{m}$-long fork-like structure formed by a transverse series of trapezoids $\left(W_{\text {in }}=500 \mathrm{~nm}, W_{\text {out }}=\right.$ $300 \mathrm{~nm})$.

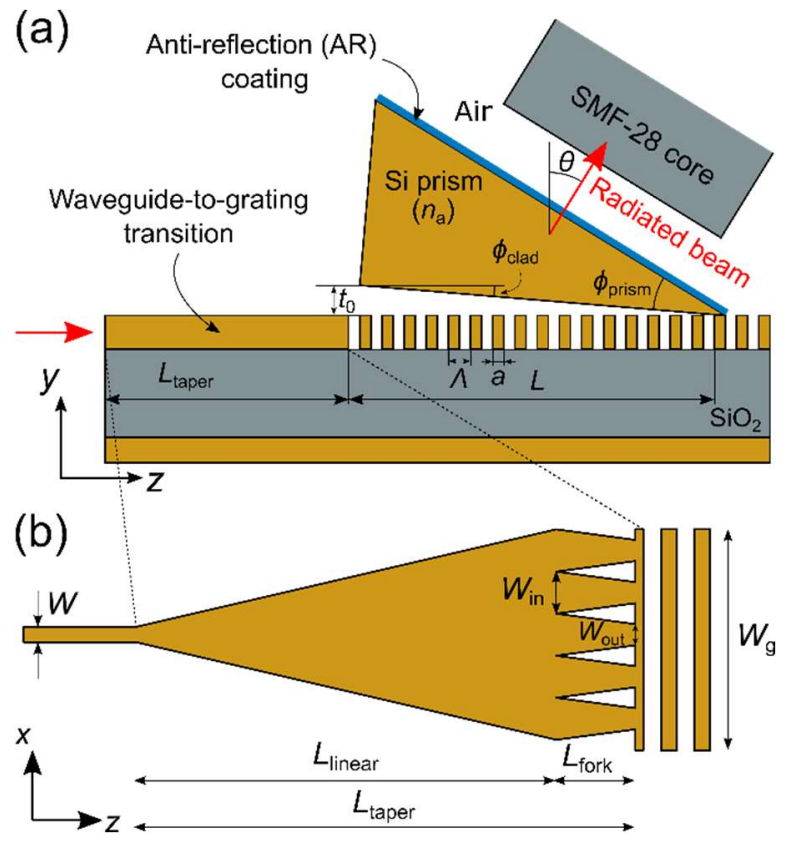

Fig. 1. (a) Schematic of a zero-order fiber-chip coupler (side view). (b) Schematic of the waveguide-to-grating transition (top view) used to adapt the guided mode of the input homogeneous waveguide to the Bloch-Floquet mode supported by the zeroorder grating coupler.

The metamaterial refractive index is engineered to minimize the effective index dispersion of the grating waveguide and therefore maximize the bandwidth. Specifically, we choose a pitch of $200 \mathrm{~nm}$ and a duty cycle of $50 \%$, which lead to a minimum feature size of $100 \mathrm{~nm}$ compatible with standard deep-ultra-violet (DUV) lithography processes. For this SWG waveguide, a theoretical coupling efficiency $>90 \%$ can be achieved by using a prism with $\phi_{\text {prism }}=35.7^{\circ}$ and length $L=20.3 \mu \mathrm{m}$, tilted $\phi_{\text {clad }}=1^{\circ}\left(t_{0}=355 \mathrm{~nm}\right)$.

Using the zero-order grating coupler, we achieve experimentally a coupling efficiency $<1 \mathrm{~dB}$ and a $1-\mathrm{dB}$ bandwidth of $94 \mathrm{~nm}$, yielding a CE-BW product exceeding $75 \mathrm{~nm}$, the highest performance yet reported for a silicon photonics surface grating coupler.

\section{Millimetre-long optical antennas with DUV-compatible feature sizes}

The rising need for compact, lightweight, and inexpensive light detection and ranging (LiDAR) systems and freespace communication transceivers has fuelled research in silicon photonics optical phased arrays (OPAs) [7,8]. Integrated optical antennas are the key components of OPAs and can be designed to enable rapid, precise, and non-mechanical steering of optical beams [9-12].

To shape high-resolution, ultra-narrow beams in the far field, grating antennas with very low radiation strengths and lengths of several millimetres are required. Achieving such long antennas in high-index-contrast platforms such as silicon on insulator makes necessary the lithographic patterning of very small perturbations (corrugations below $10 \mathrm{~nm}$ ). This requirement hinders fabrication using typical 248-nm and 193-nm DUV lithography, with fabricable minimum feature sizes between $70 \mathrm{~nm}$ and $100 \mathrm{~nm}$ [13].

We have recently reported a millimetre-long silicon optical antenna composed of an SWG waveguide core that is loaded with evanescently coupled radiative elements (see Fig. 2) [14]. The SWG metamaterial allows us to delocalize the propagating mode to accurately control the radiation strength. We choose an SWG core $W$ of $650 \mathrm{~nm}$, a period $\Lambda_{\mathrm{SWG}}$ of $170 \mathrm{~nm}$, and a duty cycle $\left(a / \Lambda_{\mathrm{SWG}}\right)$ of $\sim 53 \%$. The loading segments, of width $W_{\mathrm{s}}=$ $200 \mathrm{~nm}$ and length $L_{\mathrm{s}}=200 \mathrm{~nm}$, are separated a distance $g$ of $300 \mathrm{~nm}$ from the SWG core. Radiation is enabled by using a period $\Lambda_{\mathrm{g}}=680 \mathrm{~nm}$. Our experiments confirm a record beamwidth of $\sim 0.1^{\circ}$ for a minimum feature size of $80 \mathrm{~nm}$ and a wavelength sensitivity of $\sim 0.13^{\circ} / \mathrm{nm}$.
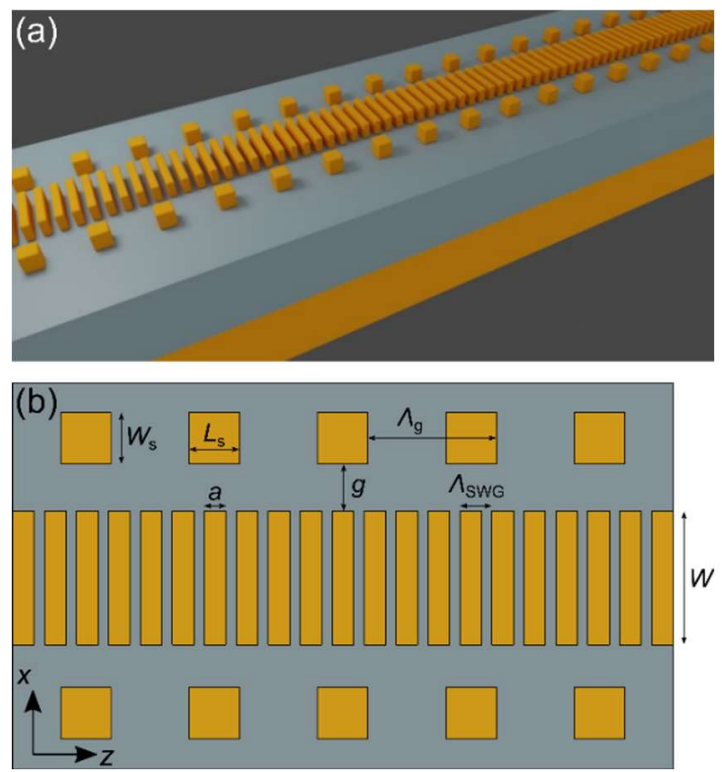

Fig. 2. Schematic (a) 3D view and (b) top view of a millimetrelong optical antenna with SWG waveguide core and evanescently coupled loading segments.

\section{Suspended germanium waveguide platform for the mid-infrared band}

The mid-infrared band has attracted great attention for important emerging applications such as biochemical 
sensing and free-space optical communications [15]. The high material losses of silicon dioxide and silicon above wavelengths of $\sim 4 \mu \mathrm{m}$ and $\sim 8 \mu \mathrm{m}$, respectively, prevent the use of the SOI platform in the longwave range. Germanium, a CMOS-compatible semiconductor that is transparent up to $\sim 15-\mu \mathrm{m}$ wavelength, can be used to develop waveguide-based devices at long wavelengths [16].

We have recently demonstrated a suspended germanium platform, based on Ge-on-SOI, operating at a wavelength of $7.7 \mu \mathrm{m}$ [17]. The waveguide is defined by etching an SWG cladding that anchors the waveguide core to lateral slabs. This cladding synthesizes a metamaterial that is judiciously engineered to provide the lateral index contrast required for light guiding. The buried silicon and silicon-dioxide layers of the Ge-on-SOI wafer are removed by a hydrofluoric (HF) acid solution, which flows through the venting holes of the lateral SWG cladding. The designed suspended waveguide has a germanium guiding layer with a nominal thickness of $1 \mu \mathrm{m}$. The core and cladding widths are $2.7 \mu \mathrm{m}$ and $3.8 \mu \mathrm{m}$, respectively, while the SWG lateral cladding has a period of $1 \mu \mathrm{m}$ and a duty cycle of $20 \%$. Figure 3 shows a scanning-electron-microscope (SEM) image of one of the fabricated suspended waveguides. At a wavelength of $7.7 \mu \mathrm{m}$, we measured a propagation loss of $5.3 \pm 1.0 \mathrm{~dB} / \mathrm{cm}$. Furthermore, by simulation we have confirmed that the proposed platform can be adapted to operate with low loss up to a wavelength of $15 \mu \mathrm{m}$.

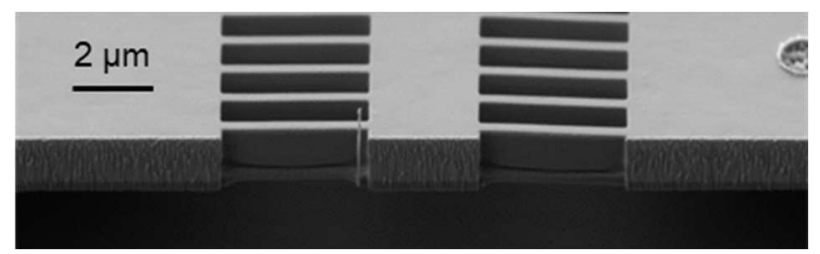

Fig. 3. SEM image of a suspended germanium waveguide designed to operate at a wavelength of $7.7 \mu \mathrm{m}$.

To couple light into the suspended germanium waveguides from a commercially available, highnumerical-aperture chalcogenide optical fibre, we utilized suspended micro-antennas [18]. These couplers exhibit a respectable coupling efficiency of $\sim 40 \%$, a broad $1-\mathrm{dB}$ spectral bandwidth in excess of $430 \mathrm{~nm}$, and a 1-dB angular bandwidth of $\sim 20^{\circ}$.

These results demonstrate the practical use of subwavelength-grating metamaterials in the longwave infrared regime.

We acknowledge funding from Universidad de Málaga, Ministerio de Ciencia, Innovación y Universidades (MCIU) (PID2019-106747RB-I00), Consejería de Economía, Conocimiento, Empresas y Universidad (CECEU) (UMA18FEDERJA-219, P18-RT-1453, P18-RT-793), and National Research Council of Canada (NRC) Collaborative Science, Technology and Innovation Program (CSTIP) (HTSN 209).

\section{References}

1. P. Cheben, R. Halir, J. H. Schmid, H. A. Atwater, and D. R. Smith, Nature 560, 565-572 (2018).
2. R. Halir, A. Ortega-Moñux, D. Benedikovic, G. Z. Mashanovich, J. G. Wangüemert-Pérez, J. H. Schmid, Í. Molina-Fernández, and P. Cheben, Proc. IEEE 106, 2144-2157 (2018).

3. J. M. Luque-González, A. Sánchez-Postigo, A. Hadij-ElHouati, A. Ortega-Moñux, J. G. Wangüemert-Pérez, J. H. Schmid, P. Cheben, Í. Molina-Fernández, and R. Halir, Nanophotonics 10, 2765-2797 (2021).

4. R. Marchetti, C. Lacava, L. Carroll, K. Gradkowski, and P. Minzioni, Photonics Res. 7, 201-239 (2019).

5. T. Tamir and S. T. Peng, Appl. Phys. 14, 235-254 (1977).

6. A. Sánchez-Postigo, R. Halir, J. G. WangüemertPérez, A. Ortega-Moñux, S. Wang, M. Vachon, J. H. Schmid, D. Xu, P. Cheben, and Í. MolinaFernández, Laser Photon. Rev. 2000542 (2021).

7. M. J. R. Heck, Nanophotonics 6, 93-107 (2017).

8. J. He, T. Dong, and Y. Xu, IEEE Access 8, 188284188298 (2020).

9. T. Kim, T. Ngai, Y. Timalsina, M. R. Watts, V. Stojanovic, P. Bhargava, C. V. Poulton, J. Notaros, A. Yaacobi, E. Timurdogan, C. Baiocco, N. Fahrenkopf, and S. Kruger, IEEE J. Solid-State Circuits 54, 3061-3074 (2019).

10. C. V. Poulton, A. Yaacobi, D. B. Cole, M. J. Byrd, M. Raval, D. Vermeulen, and M. R. Watts, Opt. Lett. 42, 4091-4094 (2017).

11. W. Ma, S. Tan, K. Wang, W. Guo, Y. Liu, L. Liao, L. Zhou, J. Zhou, X. Li, L. Liang, and W. Li, Appl. Opt. 59, 9985-9994 (2020).

12. Q. Wang, S. Wang, L. Jia, Y. Cai, W. Yue, and M. Yu, Opt. Express 29, 10509-10517 (2021).

13. M. Kamandar Dezfouli, Y. Grinberg, D. Melati, P. Cheben, J. H. Schmid, A. Sánchez-Postigo, A. Ortega-Moñux, G. Wangüemert-Pérez, R. Cheriton, S. Janz, and D.-X. Xu, Opt. Lett. 45, 3701-3704 (2020).

14. P. Ginel-Moreno, A. Sánchez-Postigo, J. De-OlivaRubio, A. Hadij-ElHouati, W. N. Ye, J. G. Wangüemert-Pérez, Í. Molina-Fernández, J. H. Schmid, P. Cheben, and A. Ortega-Moñux, Opt. Lett. 46, 3733-3736 (2021).

15. R. A. Soref, S. J. Emelett, and W. R. Buchwald, J. Opt. A Pure Appl. Opt. 8, 840-848 (2006).

16. R. Soref, Nat. Photonics 4, 495-497 (2010).

17. A. Sánchez-Postigo, A. Ortega-Moñux, J. Soler Penades, A. Osman, M. Nedeljkovic, Z. Qu, Y. Wu, I. Molina-Fernández, P. Cheben, G. Mashanovich, and J. G. Wangüemert-Pérez, Opt. Express 29, 16867-16878 (2021).

18. A. Sánchez-Postigo, A. Ortega-Moñux, D. PereiraMartín, Í. Molina-Fernández, R. Halir, P. Cheben, J. Soler Penadés, M. Nedeljkovic, G. Z. Mashanovich, and J. G. Wangüemert-Pérez, Opt. Express 27, 22302-22315 (2019). 